\title{
DINÂMICA OSCILATÓRIA EM SISTEMAS CONTENDO BROMATO E 1,4-CICLO-HEXANODIONA EM MEIO ÁCIDO. I. EFEITO DA TEMPERATURA
}

\author{
Hyrla C. L. Oliveira, Tatiane B. Oliveira e Hamilton Varela* \\ Instituto de Química de São Carlos, Universidade de São Paulo, CP 780, 13560-970 São Carlos, SP - Brasil
}

Recebido em 16/5/11; aceito em 11/8/11; publicado na web em 30/9/11

\begin{abstract}
OSCILLATORY DYNAMICS IN SYSTEMS CONTAINING BROMATE AND 1,4-CYCLOHEXANEDIONE IN ACIDIC MEDIA. I. THE EFFECT OF TEMPERATURE. We present in this work the influence of temperature on the dynamics of homogeneous chemical systems containing bromate and 1,4-cyclohexanedione (1,4-CHD) in acidic media. In particular, the following systems were studied: bromate/1,4-CHD/acid, bromate/1,4-CHD/ferroin/acid and bromate/1,4-CHD/trisbipyridine ruthenium/acid. Investigations were carried out by means of an electrochemical probe, at five temperatures between 5 and $45{ }^{\circ} \mathrm{C}$. Activation energies $\left(\mathrm{E}_{\mathrm{a}}\right)$ were estimated in different ways for the pre-oscillatory and oscillatory regimes. In any case, the $\mathrm{E}_{\mathrm{a}}$ was found to depend on the catalyst, composition and initial concentrations. In addition, it was observed that ferroin and trisbipyridine ruthenium act as catalysts only during the transition between the induction period and oscillatory regime.
\end{abstract}

Keywords: oscillations; complex kinetics; 1,4-cyclohexanedione.

\section{INTRODUÇÃO}

Uma das principais características dos sistemas vivos é a presença de ritmos nas funções regulatórias, exemplos incluem processos em diferentes níveis como os ritmos circadianos, batimentos cardíacos, ciclo menstrual, ciclo de Krebs e outros ciclos intracelulares, como a glicólise. Fenômenos desta natureza são normalmente referidos como processos de auto-organização temporal e também ocorrem em várias reações químicas na forma de oscilações nas concentrações de espécies intermediárias. Em sistemas abertos, o regime oscilatório pode persistir indefinidamente, desde que a alimentação de reagentes frescos e a remoção de produtos formados ocorram de forma adequada. Em sistemas fechados, por outro lado, as oscilações ocorrem de forma transiente e cessam quando a diminuição dos reagentes move o sistema para fora da bacia de atração referente ao estado oscilatório. Adicionalmente aos processos auto-organizados em domínio temporal, o transporte de espécies, fundamentalmente via difusão, pode cooperar com as etapas químicas envolvidas e em determinadas circunstâncias levar à formação de estruturas espaçotemporais. ${ }^{1}$ A auto-organização dinâmica ocorre em diversos sistemas físico-químicos abertos e afastados do estado de equilíbrio e vem se tornando uma área de intensa pesquisa. ${ }^{2}$

Indubitavelmente, os osciladores homogêneos baseados na redução de bromato são os mais estudados. ${ }^{3}$ A origem destes osciladores se deu na Rússia, em 1951, quando Boris Pavlovich Belousov, interessado no ciclo de Krebs, estudava a reação entre bromato e ácido cítrico em meio ácido com íons cério Ce(IV). Belousov esperava observar uma mudança na coloração da solução de amarelo para incolor, como resultado da redução do $\mathrm{Ce}(\mathrm{IV})$ para $\mathrm{Ce}(\mathrm{III})$. Em lugar de uma simples transição, a solução mudou de cor do amarelo para o incolor por diversas vezes. Belousov só conseguiu publicar sua descoberta em 1958, em um periódico bem pouco conhecido. Em 1961, Anatol M. Zhabotinskii deu continuidade ao trabalho de Belousov, e os sistemas desta classe são genericamente denominados reações de Belousov-Zhabotinskii. ${ }^{45-6}$

Atualmente são conhecidos vários sistemas químicos oscilatórios, muitos derivados da substituição e/ou da retirada de um ou

*e-mail: varela@iqsc.usp.br mais componentes presentes na formulação original do sistema BZ. Korös $^{7}$ e Orbán ${ }^{8}$ mostraram que há osciladores químicos que não exigem a presença de um catalisador metálico, os quais são chamados de sistemas BZ não catalisados ou osciladores de bromato não catalisados (uncatalyzed bromate oscillators, UBOs). ${ }^{9}$ A maioria dos UBOs utiliza em sua composição compostos aromáticos (principalmente derivados do fenol ou da anilina) como substrato orgânico e o íon bromato em meio ácido (geralmente ácido sulfúrico). ${ }^{10}$ Farage e Janjic ${ }^{11,12}$ descobriram que a 1,4-ciclo-hexanodiona (uma dicetona alicíclica) reage com o bromato em meio ácido apresentando um comportamento oscilatório. A originalidade dessa descoberta reside não apenas no fato de que o sistema oscila sem catalisador, mas no fato do fenômeno oscilatório ocorrer também em soluções de ácido sulfúrico $\left(\mathrm{H}_{2} \mathrm{SO}_{4}\right)$, ácido nítrico $\left(\mathrm{HNO}_{3}\right)$, ácido perclórico $\left(\mathrm{HClO}_{4}\right)$ e ácido fosfórico $\left(\mathrm{H}_{3} \mathrm{PO}_{4}\right)$. Os autores estudaram o efeito da temperatura, do oxigênio molecular dissolvido, do catalisador e da agitação na dinâmica oscilatória. ${ }^{11,12}$

Sabe-se que a primeira etapa da oxidação da 1,4-CHD pelo íon bromato é a abstração de um hidrogênio (processo de aromatização), em lugar da quebra da cadeia orgânica que poderia resultar, por exemplo, na formação do dióxido de carbono $\left(\mathrm{CO}_{2}\right) \cdot{ }^{10}$ Nestes estudos, verificou-se que o composto majoritário encontrado é o 1,4-di-hidroxibenzeno $\left(\mathrm{H}_{2} \mathrm{Q}\right)$ mais conhecido como hidroquinona, que é posteriormente oxidado a 1,4-benzoquinona (Q), sendo este um dos principais produtos da reação. A reação de bromação, que está sempre acompanhada da oxidação quando o íon bromato é o oxidante, tem como principais produtos, mono- e dibromo-derivados da 1,4-CHD e 1,4-bromobenzoquinona. Dessa forma, o sistema bromato/1,4-CHD/ácido despertou interesse por ser capaz de produzir ondas químicas livre de bolhas. Tal fato o torna bastante atraente uma vez que o sistema espacialmente estendido poderia ser estudado sem a perturbação decorrente do desprendimento de bolhas, em contraste ao normalmente observado na maioria das receitas tradicionais da reação $\mathrm{BZ}$, nas quais a formação de bolhas de $\mathrm{CO}_{2}$ limita sobremaneira o estudo da dinâmica espaço-temporal. A Figura 1S, material suplementar, ilustra a formação de padrões espaço-temporais no sistema $\mathrm{BrO}_{3}^{-} / 1,4-\mathrm{CHD} /$ ferroína/ácido. ${ }^{13}$ Os anéis concêntricos de cor azul claro $\left(\left[\mathrm{Fe}(\mathrm{o}-\mathrm{fen})_{3}\right]^{3+}\right.$, ferriína) se propagam em um fundo vermelho alaranjado $\left(\left[\mathrm{Fe}(\mathrm{o}-\mathrm{fen})_{3}\right]^{2+}\right.$, ferroína). A frente de onda é 
uma onda de oxidação que resulta de um aumento nas concentrações de $\mathrm{HBrO}_{2}$ e do catalisador oxidado, $\mathrm{M}_{\text {ox }}$, $\left(\left[\mathrm{Fe}(\mathrm{o}-\mathrm{fen})_{3}\right]^{3+}\right.$, ferriína $)$ e consequente queda na concentração de $\mathrm{Br}$. Enquanto que a região de cor vermelho-alaranjado corresponde à onda de redução, em que as espécies $\mathrm{HBrO}_{2}$ e $\mathrm{M}_{\mathrm{ox}}$ são consumidas e íons $\mathrm{Br}$ produzidos. Em linhas gerais, os padrões obtidos com este sistema são de boa qualidade, apresentando frentes reacionais relativamente nítidas e com um bom contraste, além da já mencionada ausência de bolhas., ${ }^{91415-16}$

Em 1998, Szalai e Körös ${ }^{9}$ propuseram um mecanismo detalhado da oxidação e bromação da 1,4-CHD pelo íon bromato em meio ácido. Em temperatura ambiente, a 1,4-CHD reage com o bromato muito lentamente. A razão para a reação ser lenta é que, ao contrário do que ocorre com os reagentes orgânicos até então utilizados, em sua maioria derivados do fenol ou da anilina, esta não é uma reação favorável por que a 1,4-CHD é simétrica e, também, porque ocorre por meio da retirada de um único hidrogênio. Portanto, é mais provável que os íons bromato ataquem as unidades $\left(-\mathrm{CH}_{2}-\mathrm{CH}_{2}-\right)$ da 1,4-CHD, produzindo um complexo ativado, não raro quando determinados oxiânions (por exemplo, $\mathrm{MnO}_{4}^{-}$ou $\mathrm{IO}_{3}^{-}$) reagem com um composto orgânico. ${ }^{9}$ A partir de estudos calorimétricos, os autores mostraram que durante o período de indução a taxa de evolução de calor é baixa e constante, indicando uma reação exotérmica lenta. ${ }^{9}$ Durante a reação, quando uma considerável quantidade de $\mathrm{H}_{2} \mathrm{Q}(10$ ${ }^{5}-10^{-4} \mathrm{~mol} \mathrm{~L}^{-1}$ ) acumula, ocorre a reação direta entre o bromato e a $\mathrm{H}_{2} \mathrm{Q}$, que, por sua vez, assume um papel fundamental nas oscilações. A 1,4-benzoquinona (Q) é formada como produto da oxidação e, em uma reação paralela, a 1,4-CHD é bromada. Em seguida, por meio da formação de BrCHD como intermediário, a 1,4-CHD é convertida a $\mathrm{H}_{2} \mathrm{Q}$ pela eliminação de $\mathrm{HBr}$. $\mathrm{O}$ íon brometo $(\mathrm{Br})$ age como inibidor até o momento que ocorre uma série de transferências de oxigênio e sua concentração cai abaixo de um valor crítico.

Indicadores de oxirredução como a ferroína $\left(\left[\mathrm{Fe}(\mathrm{o}-\mathrm{fen})_{3}\right]^{2+}\right)$ e trisbipiridinarrutênio(II) $\left(\left[\mathrm{Ru}(\mathrm{bpy})_{3}\right]^{2+}\right)$ têm sido adicionados ao sistema bromato/1,4-CHD/ácido, com o objetivo primário de tornar visível a formação de padrões espaço-temporais, como mostrado na Figura 1S, material suplementar. Como consequência, a presença de tais indicadores pode alterar significativamente a dinâmica do sistema original. Com base em um modelo prévio, ${ }^{9}$ Szalai e colaborados ${ }^{17}$ desenvolveram um mecanismo composto de 29 reações e 18 espécies para descrever o comportamento do oscilador bromato/1,4-CHD/ ferroína/ácido. Os autores verificaram que os indicadores e/ou catalisadores adicionados ao oscilador bromato/1,4-CHD/ácido participam efetivamente em ambos os processos autocatalítico e inibidor, ou ciclos de retroalimentção positivo e negativo, respectivamente; abrem um novo caminho autocatalítico; aumentam a produção de um dos principais intermediários, a hidroquinona $\left(\mathrm{H}_{2} \mathrm{Q}\right)$; e aumentam a regeneração de um intermediário de controle, o íon brometo $(\mathrm{Br})$.

A temperatura é um dos fatores externos mais importantes que afeta o comportamento dos sistemas oscilatórios. ${ }^{18}$ Em 1974, Körös ${ }^{19}$ relatou um estudo experimental do efeito da temperatura sobre oscilações na reação BZ. Körös estudou o efeito da temperatura em três sistemas oscilatórios operados em batelada: ácido malônico; bromato de potássio; ácido sulfúrico e um catalisador metálico (Ce(III), Mn(II) ou [Ru(bpy) $]^{2+}$ ). Utilizando a Equação de Arrhenius, calculou a energia de ativação $\left(\mathrm{E}_{\mathrm{a}}\right)$ utilizando a frequência do segundo ou terceiro período da oscilação e concluiu que na faixa de temperatura em que a reação foi investigada $\left(15,25\right.$ e $\left.35^{\circ} \mathrm{C}\right)$, a $\mathrm{E}_{\mathrm{a}}$ era independente da natureza do catalisador. Pastapur e Kulkarni ${ }^{20}$ estudaram o efeito da temperatura em um sistema contendo ácido oxálico, acetona, bromato, ácido sulfúrico e um catalisador (Ce(III) ou $\mathrm{Mn}(\mathrm{II})$ ), operado em batelada. Os autores chegaram a conclusões semelhantes utilizando uma faixa maior de temperatura, $25-40^{\circ} \mathrm{C}$. No entanto, Ganapathisubramanian e colaboradore ${ }^{21}$ demonstraram que nos sistemas $\mathrm{BrO}_{3}$-ácido málico envolvendo íons $\mathrm{Ce}$ (III) ou $\mathrm{Mn}$ (II) e em uma ampla faixa de temperatura $\left(25-60{ }^{\circ} \mathrm{C}\right.$, intervalo de $\left.5{ }^{\circ} \mathrm{C}\right)$, a energia de ativação para o sistema contendo íons Ce(III) foi cerca de 21-25 kJ mol ${ }^{-1}$ maior do que o observado para o sistema contendo $\mathrm{Mn}$ (II) em todos os casos.

A dependência da frequência de oscilação $(\omega)$ com a temperatura em reações BZ foi analisada por Blandamer e Roberts. ${ }^{22} \mathrm{O}$ sistema composto de ácido malônico, bromato de potássio, brometo de potássio, ácido sulfúrico e íons Ce(III) foi estudado em diferentes concentrações iniciais e em uma ampla faixa de temperatura $\left(15-68{ }^{\circ} \mathrm{C}\right)$. Os resultados indicaram que a frequência de oscilação é controlada majoritariamente pela velocidade de reação entre os íons brometo e bromato para formar $\mathrm{HOBr}$ e $\mathrm{HBrO}_{2}$. Farage e Janjic ${ }^{12}$ estudaram o efeito da temperatura $\left(15-45^{\circ} \mathrm{C}\right)$ no sistema $\mathrm{BrO}^{3-} / 1,4-\mathrm{CHD} /$ ácido $\left(\mathrm{H}_{2} \mathrm{SO}_{4}, \mathrm{HNO}_{3}, \mathrm{HClO}_{4}\right.$ e $\left.\mathrm{H}_{3} \mathrm{PO}_{4}\right)$ em batelada e verificaram que a concentração, bem como a natureza do ácido, não afetam a energia de ativação. A energia de ativação média encontrada por Farage e Janjic foi de $\mathrm{E}_{\mathrm{a}}=67 \pm 2 \mathrm{~kJ} \mathrm{~mol}^{-1}$, esse valor corrobora os valores de energia encontrados em sistemas oscilatórios catalisados ${ }^{7}$ e não catalisados. ${ }^{19,22,23}$ As diferenças observadas, segundo os autores, são devidas à natureza do substrato orgânico.

Embora o efeito da temperatura tenha sido estudado em vários sistemas BZ, o trabalho realizado por Nagy et al..$^{24}$ foi o primeiro a mostrar que a energia de ativação global é significativamente dependente do sistema BZ e de sua composição. Deve-se notar, no entanto, que no caso de mecanismos complexos, é difícil associar a energia de ativação obtida a uma única etapa do processo global. Ainda assim, uma alteração no seu valor durante o curso da reação pode indicar a passagem de um caminho cinético para outro. Embora não seja fácil encontrar o passo determinante em um estado cinético, o tipo e a complexidade das reações podem ser analisados por meio das energias de ativação obtidas. ${ }^{25}$ De acordo com Anic e colaboradores,${ }^{25}$ a energia de ativação é função da concentração dos reagentes e que mesmo em condições de concentrações constantes, dependendo do método de determinação, valores diferentes de energia de ativação podem ser obtidos.

Recentemente, o efeito da temperatura na dinâmica oscilatória do sistema bromato/hipofosfito/acetona/dois catalisadores foi investigada. Três padrões oscilatórios foram encontrados em cada temperatura, possibilitando assim estimar a energia de ativação em cada caso. Os autores traçaram a evolução temporal da energia de ativação e observaram que esta aumenta à medida que os reagentes se transformam em produtos. ${ }^{26}$ Adicionalmente aos sistemas homogêneos revisados, desenvolvimento considerável tem sido realizado no entendimento do efeito da temperatura em osciladores eletroquímicos..$^{27-30}$ Em linhas gerais, do exposto ficou claro que o entendimento dos processos resultantes da variação de temperatura em sistemas químicos oscilatórios ainda é bastante empírico e que estudos sistemáticos ainda são consideravelmente escassos.

O presente traballho foi desenvolvido com o objetivo central de estudar o efeito da temperatura na dinâmica oscilatória do sistema homogêneo bromato/1,4-CHD/ácido sulfúrico. Parâmetros como concentração do substrato orgânico, concentração do íon bromato e, a adição de catalisador também foram investigados. A seguinte notação será utilizada para descrever os sistemas derivados do oscilador-base: $\mathrm{S}_{1}$ : bromato/1,4-CHD/ácido (sem catalisador); $\mathrm{S}_{2}$ : bromato/1,4-CHD/ ferroína/ácido (em dois conjuntos de concentração diferentes, $\mathrm{S}_{2 \mathrm{a}} \mathrm{e}$ $\mathrm{S}_{2 \mathrm{~b}}$ ) e, $\mathrm{S}_{3}$ : bromato/1,4-CHD/ trisbipiridinarrutênio(II)/ácido.

\section{PARTE EXPERIMENTAL}

Os experimentos foram realizados em um reator cilíndrico de vidro de $90 \mathrm{~mL}$, com tampa de teflon. A temperatura do reator foi mantida constante por meio de um banho termostático (Marconi 


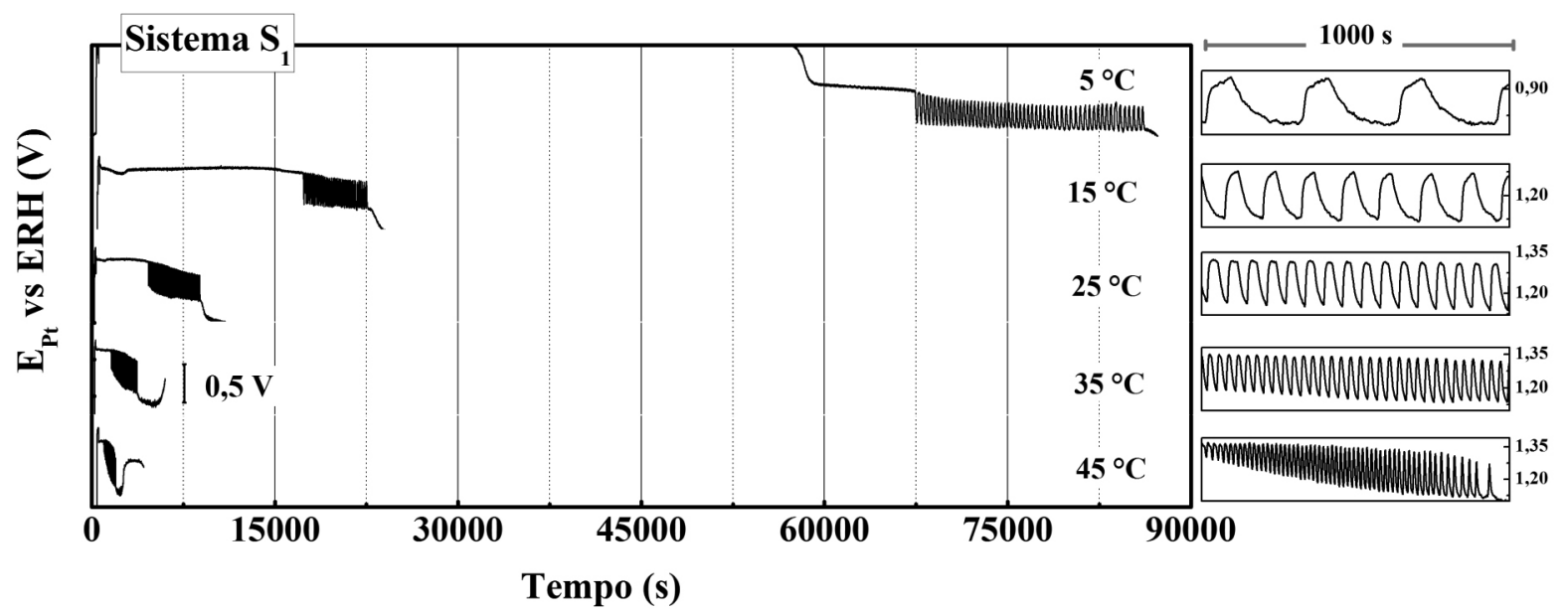

Figura 1. Evolução temporal do potencial do eletrodo de platina no sistema $S_{1}$ em todas as temperaturas estudadas. À direita são mostrados alguns detalhes das séries temporais para um mesmo intervalo de tempo e diferentes temperaturas

MA184) e o a solução mantida sob agitação constante com a utilização de um agitador magnético Marconi, modelo MA089, e uma barra magnética de teflon. O sistema reacional foi monitorado por um eletrodo de platina $\left(\mathrm{E}_{\mathrm{Pt}}\right)$ medido com relação a um eletrodo reversível de hidrogênio $(\mathrm{ERH})$ preparado na mesma concentração da solução de trabalho $\left(\left[\mathrm{H}_{2} \mathrm{SO}_{4}\right]=1,0 \mathrm{~mol} \mathrm{~L}-1\right)$. Todos os valores de potencial apresentados têm como referência o eletrodo reversível de hidrogênio.

As soluções de ferroína ([Fe(o-fen $\left.)_{3}\right]^{2+}$ ) e trisbipiridinarrutênio(II) $\left(\left[\mathrm{Ru}(\mathrm{bpy})_{3}\right]^{2+}\right)$ foram preparadas no momento da realização do experimento. Todas as soluções foram preparadas com água de alta pureza (Mili-Q, Millipore). Os reagentes: $\mathrm{H}_{2} \mathrm{SO}_{4}$, ácido sulfúrico (Mallinckrodt 97,5\%), $\mathrm{NaBrO}_{3}$, bromato de sódio (Vetec), $\mathrm{FeSO}_{4} \cdot 7 \mathrm{H}_{2} \mathrm{O}$, sulfato de ferro hepta-hidratado (Merk p.a.), $\mathrm{C}_{6} \mathrm{H}_{8} \mathrm{O}_{2}$, 1,4-ciclo-hexanodiona (Aldrich 98\%) e $\mathrm{C}_{12} \mathrm{H}_{8} \mathrm{~N}_{2}, 1,10$-fenantrolina (Riedel-de Haën p.a.) foram usados como recebidos.

Para a aquisição dos dados foi utilizado um sistema multicanal da National Instruments, modelo NI cDAQ-9172. Todos os experimentos mostrados representam resultados típicos (nas séries temporais) ou médias entre 2 ou 3 experimentos realizados.

\section{RESULTADOS E DISCUSSÃO}

Os três sistemas foram estudados com base na variação dos seguintes parâmetros: temperatura $\left(5,15,25,35\right.$ e $\left.45^{\circ} \mathrm{C}\right)$; presença ou não de um complexo metálico: sem complexo metálico, com ferroína ([Fe(o-fen $\left.)_{3}\right]^{2+}$ ) ou com o trisbipiridinarrutênio(II) ([Ru(bpy) $\left.\left.)_{3}\right]^{2+}\right)$; concentração do composto orgânico, o 1,4-ciclo-hexanodiona (1,4-CHD) e, a concentração do bromato $\left(\mathrm{NaBrO}_{3}\right)$. As concentrações iniciais utilizadas em cada sistema são ilustradas na Tabela 1.

Tabela 1. Concentrações iniciais, []$_{0}$, dos reagentes nos diferentes sistemas estudados

\begin{tabular}{cccccc}
\hline Sistema & $\begin{array}{c}\mathrm{H}_{2} \mathrm{SO}_{4} \\
\left(\mathrm{~mol} \mathrm{~L}^{-1}\right)\end{array}$ & $\begin{array}{c}\mathrm{NaBrO}_{3} \\
\left(\mathrm{~mol} \mathrm{~L}^{-1}\right)\end{array}$ & $\begin{array}{c}1,4-\mathrm{CHD} \\
\left(\mathrm{mol} \mathrm{L}^{-1}\right)\end{array}$ & $\begin{array}{c}{\left[\mathrm{Fe}(\mathrm{o}-\mathrm{fen})_{3}\right]^{2+}} \\
\left(\mathrm{mmol} \mathrm{L}^{-1}\right)\end{array}$ & $\begin{array}{c}{\left[\mathrm{Ru}(\mathrm{bpy})_{3}\right]^{2+}} \\
\left(\mathrm{mmol} \mathrm{L}^{-1}\right)\end{array}$ \\
\hline $\mathrm{S} 1$ & 1,0 & 0,10 & 0,10 & 0 & 0 \\
$\mathrm{~S}_{2 \mathrm{a}}$ & 1,0 & 0,10 & 0,10 & 0,33 & 0 \\
$\mathrm{~S}_{2 \mathrm{~b}}$ & 1,0 & 0,15 & 0,05 & 0,33 & 0 \\
$\mathrm{~S}_{3}$ & 1,0 & 0,15 & 0,05 & 0 & 0,33 \\
\hline
\end{tabular}

Oscilações estáveis em sistemas químicos ocorrem em determinadas faixas de parâmetros e dependem de uma combinação apropriada destes. Tentou-se realizar experimentos no sistema $S_{1}$ com as con- centrações de [1,4- CHD] e $\left[\mathrm{NaBrO}_{3}\right]$ iguais às do sistema $\mathrm{S}_{2 \mathrm{~b}}$, mas não foram observadas oscilações. Experimentos no sistema $S_{3}$ com concentrações iniciais de 1,4-CHD e $\mathrm{NaBrO}_{3}$ iguais às do sistema $\mathrm{S}_{1}$ não foram realizados.

Por meio das medidas do potencial de circuito aberto do eletrodo de platina no monitoramento da reação de oxidação da 1,4-CHD pelo $\mathrm{BrO}_{3}^{-}$em solução ácida, com e sem catalisador, observaram-se, após um período de indução $\left(\tau_{\text {ind }}\right)$, oscilações no potencial cujos períodos ( $\tau_{\text {ind }}$ e $\left.\tau_{\text {osc }}\right)$, amplitude $(\Delta \mathrm{E})$ e número total de oscilações $(\mathrm{N})$ diferem substancialmente a cada temperatura considerada.

A Figura 1 exemplifica a evolução temporal do potencial do eletrodo de platina $\left(\mathrm{E}_{\mathrm{Pt}}\right)$ para o sistema $\mathrm{S}_{1}$ nas cinco temperaturas estudadas. Gráficos equivalentes para os outros sistemas são mostrados no material suplementar. Em geral, oscilações de período-1 e morfologia similar foram observadas independentemente da temperatura.

\section{Período de indução}

A Figura 2 mostra o efeito da temperatura no período de indução $\left(\tau_{\text {ind }}\right)$ nos quatro sistemas estudados. Em todos os casos, o tempo de indução diminui com o aumento da temperatura. Em média, cada decréscimo de $10{ }^{\circ} \mathrm{C}$ na temperatura equivale a um aumento de três vezes no tempo de indução. Entretanto, em uma mesma temperatura são observados dois comportamentos distintos. Nas temperaturas mais altas $\left(25,35\right.$ e $\left.45^{\circ} \mathrm{C}\right)$, o sistema com trisbipiridinarrutênio(II) $\left(\mathrm{S}_{3}\right)$

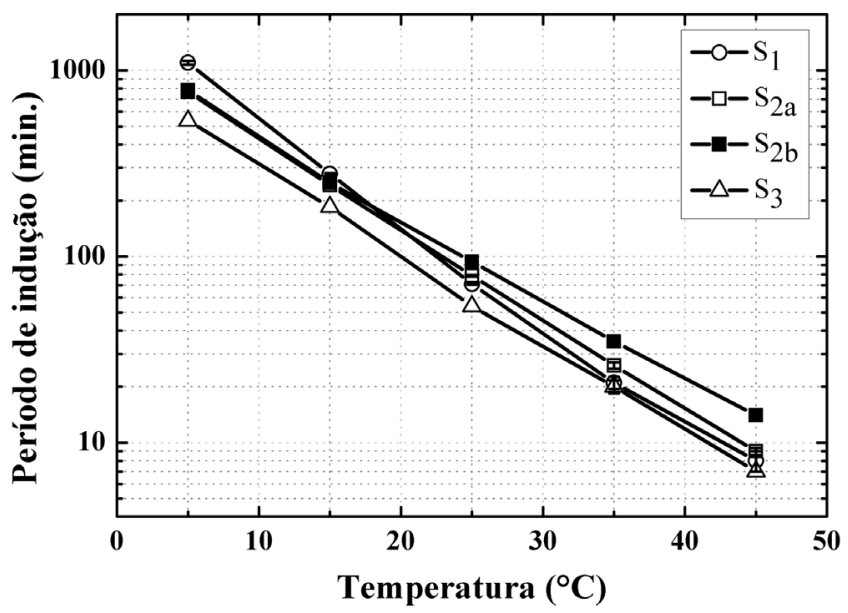

Figura 2. Efeito da temperatura no período de indução $\left(\tau_{\text {ind }}\right)$ nos quatro sistemas estudados 
apresentou o menor tempo de indução comparado com os demais, enquanto que os sistemas com ferroína $\left(\mathrm{S}_{2 \mathrm{a}}\right.$ e $\left.\mathrm{S}_{2 \mathrm{~b}}\right)$ possuem os maiores tempos de indução. Além disso, comparando os sistemas com ferroína, o $\mathrm{S}_{2 \mathrm{~b}}$ tem tempos de indução médios 1,3 vezes maiores que os do sistema $S_{2 \text { a }}$. Nas temperaturas mais baixas $\left(15\right.$ e $\left.5{ }^{\circ} \mathrm{C}\right)$, o sistema $\mathrm{S}_{3}$ continua possuindo tempo de indução menor que os demais. No entanto, os maiores tempos de indução são encontrados no sistema $S_{1}$. Nos sistemas com ferroína, o $S_{2 b}$ possui maiores tempos de indução. A partir desses resultados, pode-se ter uma visão geral de como o complexo metálico influencia o tempo de indução. Nas temperaturas estudadas, a utilização do rutênio favoreceu a diminuição do tempo de indução. No entanto, para a ferroína, o tempo de indução diminui quando em temperaturas baixas e essa diminuição é um pouco mais evidente no $\mathrm{S}_{2 \mathrm{a}}$.

Comparando os resultados mostrados na Figura 2 com resultados da literatura, constata-se que os $\tau_{\text {ind }}$ em todos os sistemas do presente trabalho são consideravelmente maiores que os observados em sistemas similares. ${ }^{12,20,21}$ Essencialmente, a diferença entre os sistemas estudados aqui, Figura 2, e outros relatados previamente pode ser relacionada ao composto orgânico. A explicação para os altos valores dos períodos de indução está na reação entre a 1,4-CHD com o $\mathrm{BrO}_{2} \bullet$, uma reação muito lenta, em contraste aos sistemas utilizando ácido oxálico ${ }^{20} \mathrm{e}$ ácido málico. ${ }^{21}$ Para que a concentração do íon brometo $\mathrm{Br}$ diminua, é necessário que uma certa quantidade de $\mathrm{H}_{2} \mathrm{Q}$ seja formada (10-5-10-4 $\left.\mathrm{mol} \mathrm{L}^{-1}\right)$. Quando isso ocorre, há a reação direta entre o bromato e a $\mathrm{H}_{2} \mathrm{Q}$. Na sequência, a 1,4- benzoquinona (Q) é formada como produto da oxidação e, em uma reação paralela, a 1,4-CHD é bromada. Por meio da formação de BrCHD como intermediário, a 1,4-CHD é convertida a $\mathrm{H}_{2} \mathrm{Q}$ pela eliminação de $\mathrm{HBr}$. O brometo age como inibidor até o momento que ocorre transferência de oxigênio e sua concentração cai abaixo de certo valor crítico. O mecanismo orgânico para a reação bromato/1,4-CHD/ácido foi proposto por Szal ai e Körös. ${ }^{9}$

Na Figura 2 também é possível observar um ponto de inversão de comportamento (em torno de $20^{\circ} \mathrm{C}$ ) nos sistemas $\mathrm{S}_{1}, \mathrm{~S}_{2 \mathrm{a}}$ e $\mathrm{S}_{2 \mathrm{~b}}$. A mesma inversão acontece no sistema estudado por Ganapathisubramanian e colaboradores ${ }^{21}{ }^{21}$ no entanto, em uma temperatura mais alta (em torno de $35^{\circ} \mathrm{C}$ ). $\mathrm{O} \tau_{\text {ind }}$ observado a $35^{\circ} \mathrm{C}$ para o sistema $\mathrm{S}_{1}$ é bem próximo ao valor obtido por Farage e Janjic ${ }^{12}$ em seu trabalho com o mesmo sistema nas mesmas concentrações. No presente trabalho, em todos os sistemas, o logaritmo do $\tau_{\text {ind }}$ varia linearmente com a temperatura.

\section{Período oscilatório}

O período em que o sistema passa oscilando $\left(\tau_{\text {osc }}\right)$, a amplitude $(\Delta \mathrm{E})$ e o número $(\mathrm{N})$ de oscilações são fatores que podem fornecer informações importantes sobre a dinâmica oscilatória quando se varia a temperatura. A Figura 3 mostra o período oscilatório em função da temperatura nos três sistemas estudados. Inicialmente percebe-se que os sistemas estudados passam mais tempo oscilando que os demais sistemas BZ encontrados na literatura, ${ }^{20,21,24,31}$ até mesmo quando comparados a um sistema de igual composição e concentração inicial. ${ }^{12} \mathrm{~A}$ segunda observação a ser feita refere-se à considerável diferença encontrada entre os valores de $\tau_{\text {osc }}$ para o $S_{1}$ e o sistema de igual composição, concentração e condições experimentais relatado na literatura. ${ }^{12}$ Essa diferença não foi evidenciada no $\tau_{\text {ind }}\left(\tau_{\text {ind }}=21\right.$ min, presente trabalho; $\tau_{\text {ind }}=24$ min na ref. 12).

Outras informações podem ser extraídas acerca do efeito da temperatura no período oscilatório a partir da Figura 3: exceto o sistema anteriormente estudado por nosso grupo, ${ }^{26}$ apenas o estudado por Ganapathisubramaniam e colaboradore ${ }^{21}$ possui períodos oscilatórios próximos aos observados aqui; em nenhum dos sistemas estudados no presente trabalho, o logaritmo do $\tau_{\mathrm{osc}}$ em função da temperatura possui

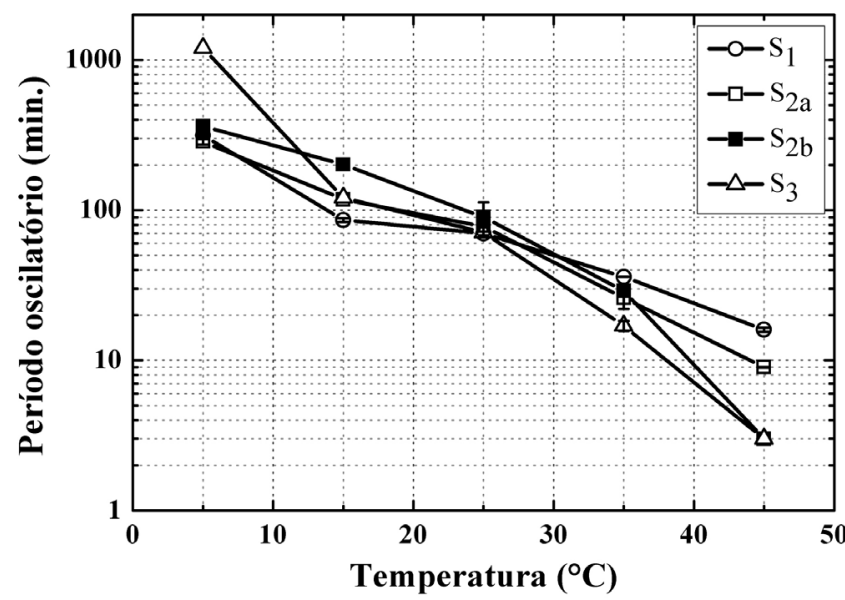

Figura 3. Efeito da temperatura no período oscilatório $\left(\tau_{o s c}\right)$ nos quatro sistemas estudados

perfil linear, como encontrado na literatura; ${ }^{12,20,26,31} \mathrm{e}$, finalmente, o fato de que mesmo tendo sido operado em regime de fluxo contínuo (CSTR - continuous-flow stirred-tank reactor), o sistema estudado por Basavaraja e Kulkarni exibe período semelhante aos demais, operados em batelada. ${ }^{31}$ De maneira geral, comparando os sistemas estudados no presente trabalho com os da literatura ${ }^{20,21}$ tem-se que aqueles passam mais tempo oscilando, apesar de levarem um tempo maior para atingirem o regime oscilatório.

\section{Amplitude das oscilações}

As amplitudes médias $(\Delta \mathrm{E})$ das oscilações no potencial do eletrodo de platina em função da temperatura para os três sistemas estudados também foram avaliadas. Comparando os sistemas, dois comportamentos se destacam - o $\mathrm{S}_{2 \mathrm{a}}$ e o $\mathrm{S}_{2 \mathrm{~b}}$ possuem perfis semelhantes e os sistemas $S_{1}$ e $S_{3}$ possuem perfis completamente distintos. Com relação aos sistemas $S_{2 a}$ e $S_{2 b}$, destaca-se o valor de $\Delta E=2,6$ vezes maior do sistema $S_{2 a}$ em relação ao $S_{2 b}$ observado em quase toda a faixa de temperatura. Outro fato interessante está na temperatura coincidente em que foi encontrado o maior valor de $\Delta \mathrm{E}$ em ambos os sistemas, $25^{\circ} \mathrm{C}$. Em um sistema estudado por Basavaraja e Kulkarni, ${ }^{31}$ o maior valor de $\Delta \mathrm{E}$ também foi na temperatura de $25^{\circ} \mathrm{C}$, no entanto, quando comparado ao $S_{2 a}$ esse valor é menor. Os sistemas $S_{1}$ e $S_{3}$, por outro lado, exibiram comportamentos completamente distintos. Os maiores valores de $\Delta \mathrm{E}$ para o $\mathrm{S}_{1}$ estão nas temperaturas de $15 \mathrm{e}$ $35^{\circ} \mathrm{C}$, exatamente onde estão os menores valores de $\Delta \mathrm{E}$ para o $\mathrm{S}_{3}$ e os maiores valores de $\Delta \mathrm{E}$ para o $\mathrm{S}_{3}$, nas temperaturas de 5 e $45^{\circ} \mathrm{C}$, estão exatamente onde se encontram os menores valores de $\Delta \mathrm{E}$ para o $\mathrm{S}_{1}$.

$\mathrm{O}$ valor médio de $\Delta \mathrm{E}$ encontrado na temperatura de $35{ }^{\circ} \mathrm{C}$ no $\mathrm{S}_{1}$ é 1,6 vezes superior ao valor obtido previamente em um sistema estudado sob condições experimentais, composição e concentração inicial semelhantes. ${ }^{12}$ Apesar dos perfis diferentes, os sistemas $S_{1}$ e $\mathrm{S}_{2 \mathrm{a}}$, bem como os sistemas $\mathrm{S}_{2 \mathrm{~b}}$ e $\mathrm{S}_{3}$, se assemelham nos valores médios de $\Delta \mathrm{E}$. Vale ressaltar que as maiores amplitudes obtidas com o presente trabalho estão nos sistemas $\mathrm{S}_{1}$ e $\mathrm{S}_{2 \mathrm{a}}(\sim 150 \mathrm{mV})$. Contudo, o sistema estudado por Nogueira et al. ${ }^{26}$ apresentou maiores amplitudes $(\sim 190 \mathrm{mV})$.

\section{Número de oscilações}

A Figura 4 exibe o número de oscilações $(\mathrm{N})$ em função da temperatura para os três sistemas estudados. Percebe-se inicialmente que, diferentemente do que foi visto para tempo de indução, período oscilatório e amplitude, os sistemas que contêm ferroína, $S_{2 \mathrm{a}} \mathrm{e}$ 


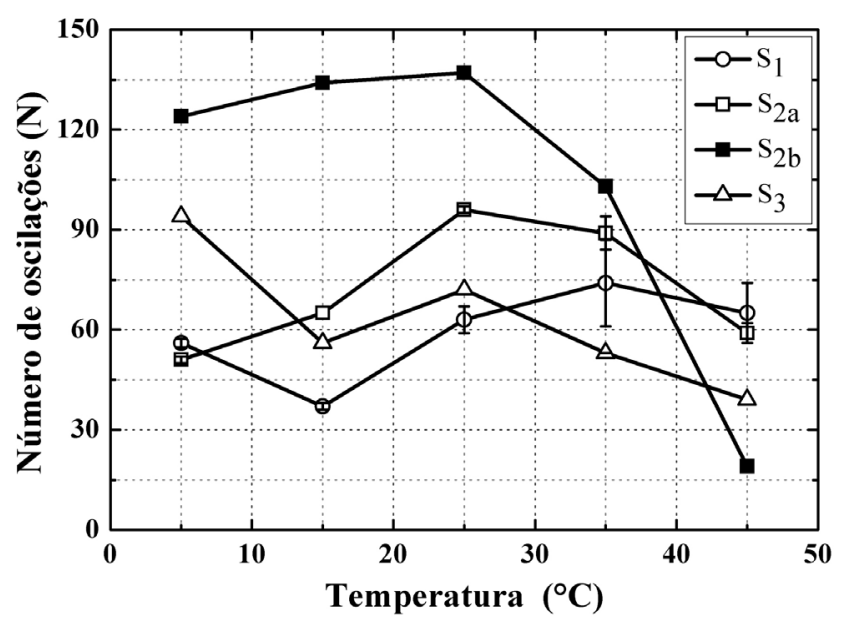

Figura 4. Efeito da temperatura no número de oscilações $(N)$ nos quatro sistemas estudados

$\mathrm{S}_{2 \mathrm{~b}}$, possuem perfis distintos. No sistema estudado por Nogueira e colaboradores, ${ }^{26} \mathrm{o}$ número de oscilações aumenta à medida que a temperatura aumenta. Em $35{ }^{\circ} \mathrm{C}$ o sistema atinge seu maior número de oscilações apesar de ainda ser o menor em relação aos demais sistemas na mesma temperatura. Nos sistemas $S_{2 a}$ e $S_{2 b}$, o aumento do número de oscilações com o aumento da temperatura ocorre até os $25^{\circ} \mathrm{C}$; após essa temperatura, o $\mathrm{N}$ cai. Outro fato que se destaca é o comportamento do $\mathrm{S}_{2 b}$. A $5{ }^{\circ} \mathrm{C}$ o sistema possui o maior $\mathrm{N}$ e quando chega aos $45^{\circ} \mathrm{C}$ atinge o menor $\mathrm{N}$ em relação aos outros. Mais uma vez o sistema de composição, concentração e condições experimentais semelhantes, estudado por Farage e Janjic, ${ }^{12}$ difere do $S_{1}$.

Os resultados indicam que os sistemas $S_{2 a}$ e $S_{2 b}$ são mais robustos que os $S_{1}$ e $S_{3}$, uma vez que quanto maior o número de oscilações, maior é a estabilidade do oscilador.

\section{Energia de ativação}

Analisando a influência da temperatura em todos os sistemas estudados, verificou-se que o gráfico nas coordenadas de Arrhenius ( $\ln \omega$ vs 1/RT) é linear dentro da faixa de temperatura investigada, com um bom grau de correlação $(\mathrm{R}>0,98)$. Assim, é conveniente caracterizar a dependência da temperatura por meio dos valores da energia de ativação $\left(E_{a}\right)$. Da mesma forma, a influência da temperatura no tempo de indução foi examinada, ainda nas coordenadas de Arrhenius (ln 1/ $\tau_{\text {ind }}$ vs. 1/RT), o que também fornece uma dependência linear na faixa de temperatura estudada (Figura 5). Uma vez que as séries temporais mostraram que as frequências $(\omega)$ das oscilações variam ao longo do tempo, foi necessário avaliar qual região seria a mais apropriada, ou qual região representaria melhor a reação global para efeito do cálculo da energia de ativação. Körös ${ }^{19}$ justifica as variações nas frequências ao longo do tempo como resultado das mudanças nas concentrações dos reagentes. De fato, convém enfatizar que todos os experimentos apresentados aqui foram realizados em batelada.

Por considerar as oscilações químicas como uma série de reações autocatalíticas "explosivas" ocorrendo com certa frequência, Körös ${ }^{19}$ concluiu que a energia de ativação não depende da natureza do catalisador nem da concentração, pelo menos na faixa em que foi considerada. Em 1991, Pastapur e Kulkarni ${ }^{20}$ calcularam a energia de ativação para sistemas com dois substratos orgânicos (ácido sulfúrico/ bromato/ácido oxálico/acetona/Mn(II) ou Ce(III)) e concluíram que a energia de ativação para estes sistemas, na faixa de concentração estudada, não depende da natureza do catalisador. No entanto, nesse mesmo trabalho, ${ }^{20}$ os autores relatam que a abordagem utilizada
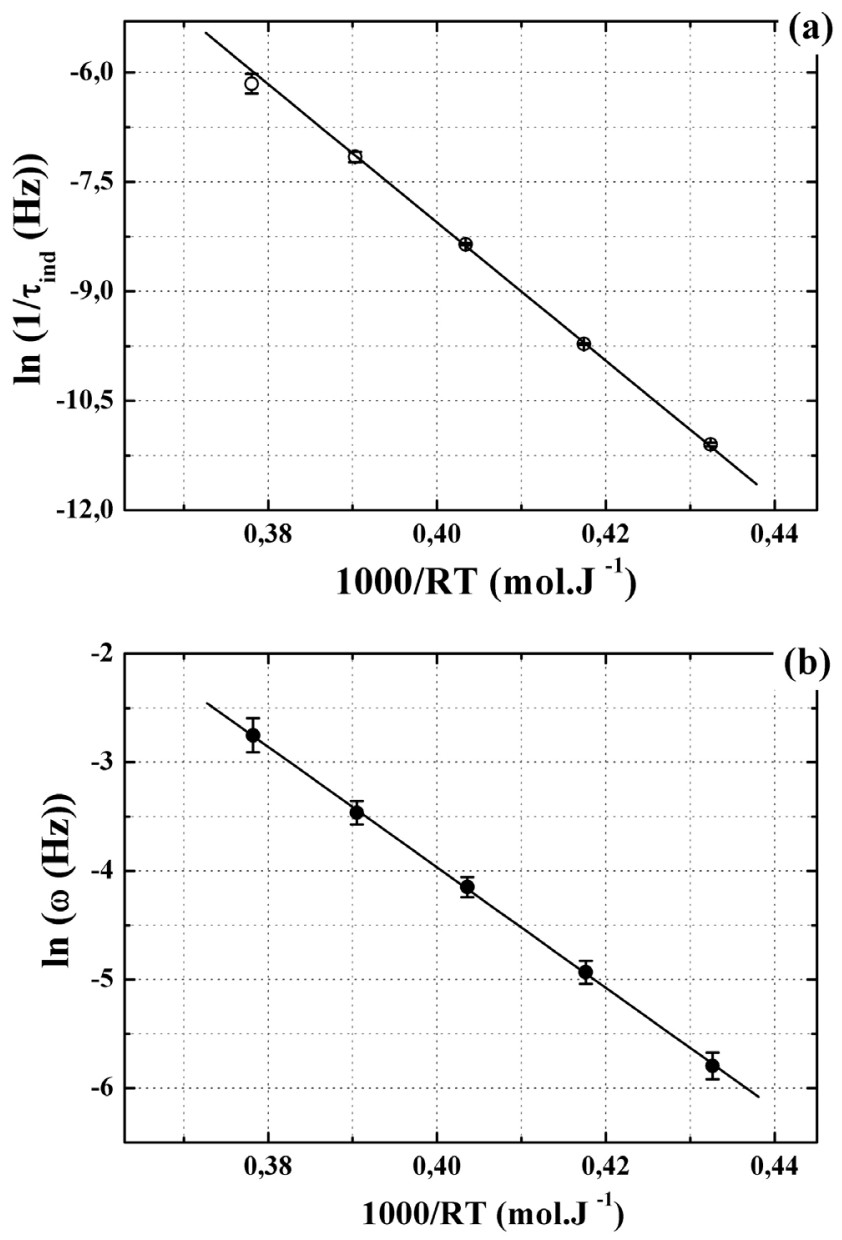

Figura 5. Gráfico nas coordenadas de Arrhenius para o sistema $S_{l}$ : (a) $\ln \left(1 / \tau_{\text {ind }}\right)$ vs $1 / R T(R=0,99968)$; (b) $\ln (\omega)$ vs $1 / R T(R=0,99982)$

tomou como base um tratamento monomolecular, mas que o sistema como um todo envolve uma série de etapas. Pastapur e Kulkarni ${ }^{20}$ consideraram que, ainda que seja possível que as reações autocatalíticas que levam à produção de bromo e espécies metálicas oxidadas sejam semelhantes, a parte final da reação onde existem oscilações de períodos diferentes do início da reação pode envolver um consumo de bromo e de espécies metálicas oxidadas diferentes. Adicionalmente, os autores vão além quando afirmam que um melhor entendimento poderia ser alcançado se a energia de ativação fosse calculada para vários passos elementares, como realizado por Kumpinsky e Epstein. ${ }^{32}$

Ganapathisubramanian e colaboradore ${ }^{21}$ obtiveram a energia de ativação para o processo global da reação, utilizando também a Equação de Arrhenius e com períodos de oscilações do início da reação. O sistema era composto de ácido sulfúrico, ácido málico, bromato, $\mathrm{Mn}(\mathrm{II})$ ou Ce(III). Os autores verificaram que a energia de ativação do sistema contendo íons cério é maior $\left(21 \mathrm{a}^{2} \mathrm{~kJ} \mathrm{~mol}^{-1}\right)$ que para o sistema contendo manganês em todos os casos e considerando os diferentes tempos $\left(\tau_{\text {ind }}\right.$ ou $\left.1 / \omega\right)$.

O trabalho de Nagy et al. ${ }^{24}$ foi provavelmente o primeiro a mostrar que a energia de ativação é significativamente dependente do sistema oscilatório em questão e sua composição. Os autores alegaram que a frequência de oscilação era controlada pela velocidade de reação entre íons brometo e íons bromato para formar ácido hipobromoso e ácido bromoso, e que outras reações que consomem íons brometo deveriam ser consideradas no cálculo da energia de ativação. Anic e colaboradores ${ }^{25}$ calcularam a energia de ativação na reação de BrayLiebhafsky. Os autores relataram que mesmo que as concentrações 
iniciais dos reagentes sejam constantes, dependendo do método de determinação, diferentes valores de energia de ativação podem ser obtidos. Assim, os autore ${ }^{25}$ sugeriram seis métodos diferentes para o cálculo das energias de ativação na reação de decomposição do peróxido de hidrogênio.

Como já mencionado, Nogueira et al. ${ }^{26}$ verificaram a existência de três padrões oscilatórios sequenciais em cada temperatura investigada. Baseados nos diferentes padrões oscilatórios estimaram a energia de ativação em diferentes momentos da reação. Como a morfologia das séries relatadas aqui não varia de forma clara, optouse por um método alternativo de se avaliar o efeito da passagem do tempo na dinâmica do sistema. Uma maneira encontrada para tal avaliação foi colocar os períodos de oscilação em função do tempo, em cada sistema e temperatura estudados, como mostrado na Figura 4S, material suplementar. Procedimento similar foi adotado por Dutt e Banerjee. ${ }^{33}$

A Figura 6 mostra a evolução temporal do período de cada oscilação $i$ no sistema $\mathrm{S}_{1}$ nas cinco temperaturas estudadas. Percebe-se claramente a presença de três regiões distintas, na forma de $U$. A adição do catalisador, no entanto, modifica este padrão, como ilustrado nas Figuras 4S e 5S, material suplementar. A primeira região (região I) corresponde à transição de um regime estacionário para um regime oscilatório. A segunda região (região II) corresponde a um regime oscilatório intermediário com período aproximadamente constante.

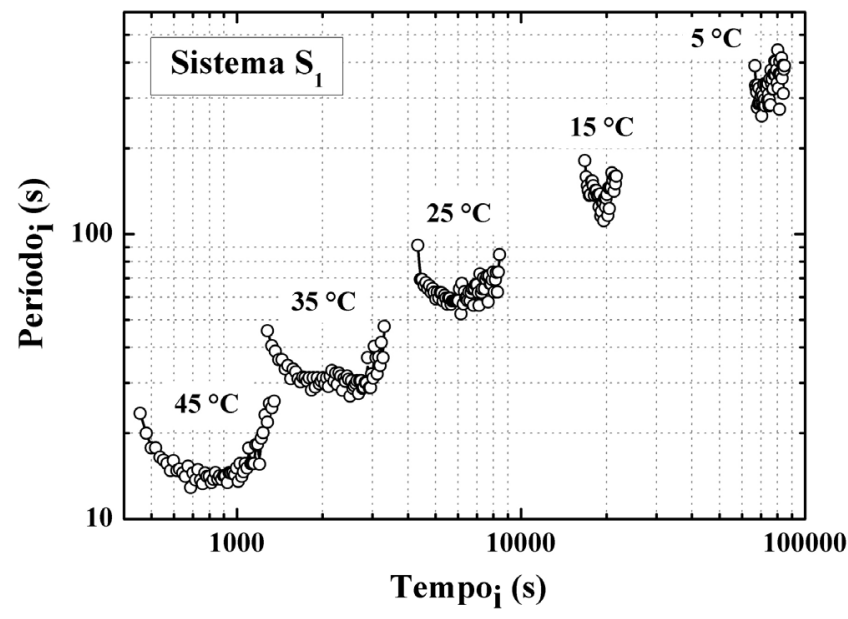

Figura 6. Evolução temporal do período das oscilações no sistema $S_{1}$ a diferentes temperaturas

A terceira região corresponde ao final do regime oscilatório e coincide com um aumento do período oscilatório. Experimentalmente não foi possível distinguir de maneira precisa se as oscilações cessam por meio de uma bifurcação subcrítica de Hopf ou de forma homoclínica. Em razão da natureza dessas três etapas descritas anteriomente, o gráfico nas coordenadas período e tempo adquire a forma de $U$. A adição do catalisador ao sistema parece favorecer uma transição mais rápida entre as regiões I e II, de modo que o período se modifica e a curva parece perder o ramo da região I.

Partiu-se então da suposição de que cada região anteriormente descrita pode se caracterizar por processos distintos. Vale ressaltar mais uma vez que não se visualizou, nas séries temporais, uma mudança evidente na forma das oscilações e que a definição das regiões seguiu o esquema mostrado na Figura 6. Além da frequência média de cada região $(\omega)$ também se utilizou o tempo de indução $\left(\tau_{\text {ind }}\right)$ no cálculo da energia de ativação. Na Figura 7 pode-se observar a evolução temporal da energia de ativação nas quatro regiões ( $\tau_{\text {ind }}$; regiões I, II e III). O cálculo da energia de ativação também foi feito seguindo o procedimento que vários autores ${ }^{11,19-21,24}$ utili-

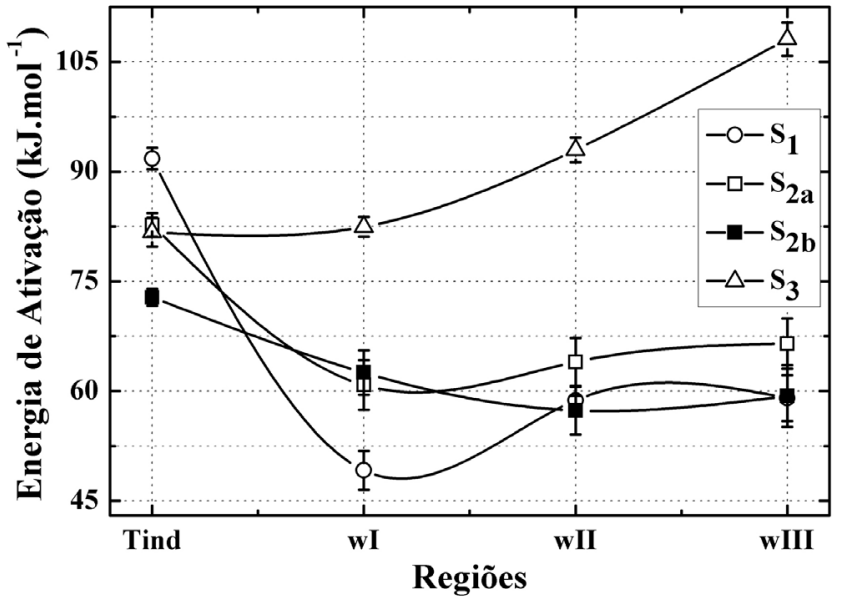

Figura 7. Evolução temporal da energia de ativação para os quatro sistemas estudados, para o tempo de indução $\left(T_{\text {ind }}\right)$ e as três sucessivas regiões (wI, $w I I$ e wIII)

zaram, por meio do período da segunda oscilação $\omega_{2 \text { nd }}$, ver material suplementar.

Baseado nos resultados obtidos pode-se inferir que a energia de ativação é dependente do tipo de catalisador utilizado, bem como da composição e concentrações iniciais, diferentemente do que foi afirmado por Körös ${ }^{19}$ e Pastapur e Kulkarni. ${ }^{20}$ Analisando inicialmente o período de indução, percebe-se que a adição do catalisador favorece a diminuição da $\mathrm{E}_{\mathrm{a}}$ e essa diminuição é mais pronunciada para o sistema $\mathrm{S}_{2 \mathrm{~b}}$. No entanto, a $\mathrm{E}_{\mathrm{a}}$ aumenta nas regiões oscilatórias e o aumento é maior no sistema $\mathrm{S}_{3}$ em todas as regiões oscilatórias. Comparando os sistemas que possuem ferroína, pode-se perceber que o $\mathrm{S}_{2 b}$ é o sistema que possui menor $\mathrm{E}_{\mathrm{a}}$ em todas as regiões. Em comparação a outros sistemas BZ relatados na literatura, percebemse diferenças significativas, mesmo quando se comparam sistemas de igual composição, concentração e condições experimentais como no caso do sistema $\mathrm{S}_{1}, \operatorname{com} \mathrm{E}_{\mathrm{a}}=51 \mathrm{~kJ} \mathrm{~mol}^{-1}$, utilizando o período da segunda oscilação $\left(\omega_{2 n d}\right)$ e o relatado por Farage e Janjic, ${ }^{12} \mathrm{E}_{\mathrm{a}}=70 \mathrm{~kJ}$ $\mathrm{mol}^{-1}$, também utilizando o período da segunda oscilação no cálculo.

Considerando os resultados obtidos por meio do cálculo da energia de ativação e a definição de catalisador como uma substância que acelera uma reação, diminuindo a energia de ativação e a energia do complexo ativado, sem ser consumido, durante o processo, observa-se que a ferroína $\left(\left[\mathrm{Fe}(\mathrm{o}-\mathrm{fen})_{3}\right]^{2+}\right)$ e o trisbipiridinarrutênio(II) $\left(\left[\mathrm{Ru}(\mathrm{bpy})_{3}\right]^{2+}\right)$ agem de forma efetiva, promovendo um mecanismo molecular diferente para a reação, na transição do período de indução para o início da região oscilatória. É nessa região em que quase todos os sistemas estudados, com exceção do $\mathrm{S}_{3}$, que a energia de ativação tem seu valor diminuído. Em outros sistemas $\mathrm{BZ},{ }^{12,20,21}$ a $\mathrm{E}_{\mathrm{a}}$ é maior no início da reação. Esse valor, como no presente trabalho, decresce durante o período de indução até o momento em que a reação começa a oscilar. Vale ressaltar que essa dedução é baseada na observação do comportamento global. Tal fato não exclui a possibilidade dos complexos metálicos agirem como catalisadores nas várias etapas (às quais não temos acesso) que compõem uma reação oscilatória.

Na tentativa de verificar o método escolhido na divisão de regiões e a possibilidade de distinguir processos diferentes que possam ocorrer durante a reação (quando não é possível a visualização de padrões oscilatórios), calcularam-se as energias de ativação do sistema estudado por Nogueira et al. ${ }^{26}$ utilizando a curva do período em função do tempo. Os resultados mostram que, apesar do erro no ajuste (regressão linear) ser relativamente grande, o método proposto é útil para distinguir processos paralelos durante a reação. 


\section{CONCLUSÕES}

Foram apresentados neste trabalho resultados experimentais do efeito da temperatura (cinco temperaturas entre 5 e $45^{\circ} \mathrm{C}$ ) sobre a dinâmica oscilatória dos sistemas bromato/1,4-CHD/ácido $\left(\mathrm{S}_{1}\right)$, bromato/1,4-CHD/ferroína/ácido ( $\mathrm{S}_{2}$, em duas composições distintas, $\mathrm{S}_{2 \mathrm{a}}$ e $\mathrm{S}_{2 \mathrm{~b}}$ ) e bromato/1,4-CHD/trisbipiridinarrutênio(II) /ácido $\left(\mathrm{S}_{3}\right)$. A partir das séries temporais foi possível avaliar como a temperatura influencia parâmetros importantes, tais como período de indução $\left(\tau_{\text {ind }}\right)$, período oscilatório $\left(\tau_{\text {osc }}\right)$, amplitude $(\Delta \mathrm{E})$, número de oscilações $(\mathrm{N})$ e energia de ativação $\left(\mathrm{E}_{\mathrm{a}}\right)$.

Com base na literatura observou-se que os altos valores dos períodos de indução para todos os sistemas estudados se devem ao fato da reação entre a 1,4-CHD e o $\mathrm{BrO}_{2} \bullet$ ser desfavorável. Em temperaturas mais altas $\left(25,35\right.$ e $\left.45^{\circ} \mathrm{C}\right)$, o sistema com rutênio, $S_{3}$, possui menor tempo de indução comparado com os demais sistemas, enquanto que os sistemas contendo ferroína $\left(\mathrm{S}_{2 \mathrm{a}}\right.$ e $\left.\mathrm{S}_{2 \mathrm{~b}}\right)$ possuem os maiores tempos de indução. Nas temperaturas estudadas, a utilização do complexo de rutênio favoreceu a diminuição do tempo de indução. No entanto, em sistemas contendo ferroína, o tempo de indução diminui quando em temperaturas baixas. Com relação ao período oscilatório, os valores encontrados neste trabalho são maiores que aqueles obtidos para a maioria dos sistemas BZ relatados na literatura, ainda que se comparem sistemas de mesma composição, concentração inicial e condições experimentais.

Ao se avaliar o efeito da temperatura sobre a amplitude das oscilações, dois comportamentos se destacam. Os sistemas $\mathrm{S}_{2 \mathrm{a}}$ e $\mathrm{S}_{2 \mathrm{~b}}$ possuem perfis semelhantes e os sistemas $\mathrm{S}_{1}$ e $\mathrm{S}_{3}$ possuem comportamentos totalmente opostos. As inversões de comportamento vistas nos períodos de indução e oscilatório e na amplitude também são encontradas quando se analisa o número de oscilações do sistema. Em todos os sistemas estudados o gráfico nas coordenadas de Arrhenius é linear dentro da faixa de temperatura investigada, com um bom grau de confiabilidade. Como consequência, pode-se analisar a dependência da temperatura por intermédio dos valores da energia de ativação. As energias de ativação foram obtidas de três formas: seguindo o procedimento utilizado por diversos autores (frequência da segunda oscilação); utilizando a frequência média da região oscilatória e, por meio da divisão em três regiões do gráfico período versus tempo. Em todos os casos, a energia de ativação é dependente do tipo de catalisador utilizado, bem como da composição e concentrações iniciais do sistema. Verificouse ainda que, globalmente, a $\left(\left[\mathrm{Fe}(\mathrm{o}-\mathrm{fen})_{3}\right]^{2+}\right)$ e o $\left(\left[\mathrm{Ru}(\mathrm{bpy})_{3}\right]^{2+}\right)$ atuam como catalisadores durante a transição do período de indução para o período oscilatório, uma vez que é durante esse período que a energia de ativação decresce, em quase todos os sistemas estudados. Contudo, não se pode afirmar que nas regiões oscilatórias estes não atuem como catalisadores, uma vez que o comportamento global da região oscilatória é resultado da combinação de várias etapas.

\section{MATERIAL SUPLEMENTAR}

Contendo Figuras 1S a 7S encontra-se disponível em http://quimicanova.sbq.org.br, na forma de arquivo PDF, com acesso gratuito.

\section{AGRADECIMENTOS}

Os autores agradecem a G. Metzker e ao Prof. D. Cardoso pela síntese do complexo de rutênio. H. C. L. Oliveira agradece ao Con- selho Nacional de Desenvolvimento Científico e Tecnológico (CNPq, \# 142727/2006-7) pela bolsa concedida. T. B. Oliveira agradece à Fundação de Amparo à Pesquisa do Estado de São Paulo (FAPESP) pela bolsa concedida (\# 2008/08198-6). H. Varela agradece à FAPESP (\# 2004/04528-0 e \# 2009/07629-6) e ao CNPq (\# 302698/2007-8 e \# 306151/2010-3) pelos auxílios concedidos.

\section{REFERÊNCIAS}

1. Nicolis, G.; Prigogine, I.; Self-Organization in Nonequilibrium Systems: from Dissipative Structures to order through Fluctuations, John Wiley \& Sons: New York, 1977.

2. Varela, H.; Ciência e Cultura 2011, 63, 23.

3. Sagues, F.; Epstein, I. R.; Dalton Trans. 2003, 1201.

4. Zhabotinsky, A. M.; Chaos 1991, 1, 379.

5. Faria, R. B.; Quim. Nova 1995, 18, 281.

6. Zhabotinsky, A. M.; Scholarpedia 2007, 2, 1435.

7. Körös, E.; Orbán, M.; Nature 1978, 273, 371.

8. Orbán, M.; Körös, E.; J. Phys. Chem. 1978, 82, 1672.

9. Szalai, I.; Körös, E.; J. Phys. Chem. A 1998, 102, 6892.

10. Kurin-Csörgei, K.; Szalai, I.; Molnár-Perl, I.; Körös, E.; React. Kinet. Catal. Lett. 1994, 53, 115.

11. Farage, V. J.; Janjic, D.; Chem. Phys. Lett. 1982, 88, 301.

12. Farage, V. J.; Janjic, D.; Chem. Phys. Lett. 1982, 93, 621.

13. Oliveira, H. C. L.; Tese de Doutorado, Universisdade de São Paulo, Brasil, 2011.

14. Kurin-Csörgei, K.; Szalai, I.; Körös, E.; React. Kinet. Catal. Lett. 1995, 54, 217.

15. Britton, M. M.; J. Phys. Chem. A 2003, 107, 5033.

16. Kurin-Csörgei, K.; Zhabotinsky, A. M.; Orbán, M.; Epstein, I.R.; J. Phys. Chem. 1996, 100, 5393.

17. Szalai, I.; Kurin-Csörgei, K.; Orbán, M.; Phys. Chem. Chem. Phys. 2002, $4,1271$.

18. Misra, G. P.; Chem. Phys. Lett. 1992, 191, 435.

19. Körös, E.; Nature 1974, 251, 703.

20. Pastapur, S. M.; Kulkarni, V. R.; J. Ind. Chem. Soc. 1991, 68, 293.

21. Ganapathisubramanian, N.; Jaya, S.; Ramaswamy, R.; Curr. Sci. 1978, 47,770 .

22. Blandamer, M. J.; Roberts, D. L.; J. Chem. Soc. Faraday Trans. 1977, 73,1056

23. Burger, M.; Körös, E. ; J. Phys. Chem. 1980, 84, 496.

24. Nagy, G.; Körös, E.; Oftedal, N.; Tjelflaat, K.; Ruoff, P.; Chem. Phys. Lett. 1996, 250, 255.

25. Anic, S.; Kolar-Anic, L.; Körös, E.; React. Kinet. Catal. Lett. 1997, 61, 111.

26. Nogueira, P. A.; Oliveira, H. C. L.; Varela, H.; J. Phy. Chem. A $\mathbf{2 0 0 8 , ~}$ $112,12412$.

27. Nagao, R.; Epstein, I. R.; Gonzalez, E. R.; Varela, H.; J. Phys. Chem. A 2008, $112,4617$.

28. Angelucci, C. A.; Varela, H.; Herrero, E.; Feliu, J.; J. Phys. Chem C 2009, 113, 18835.

29. Carbonio, E. A.; Nagao, R.; Gonzalez, E. R.; Varela, H.; Phys. Chem. Chem. Phys. 2009, 11, 665.

30. Sitta, E.; Nascimento, M. A.; Varela, H.; Phys. Chem. Chem. Phys. 2010, $12,15195$.

31. Basavaraja, C.; Kulkarni, V. R.; J. Ind. Chem. Soc. 2004, 81, 427.

32. Kumpisnky, E.; Epstein, I. R.; J. Phys. Chem. 1985, 89, 688.

33. Dutt, A. K.; Banerjee, R. S.; Chem. Phys. Lett. 1983, 99, 186. 